\title{
White South or Central American
}

National Cancer Institute

\section{Source}

National Cancer Institute. White South or Central American. NCI Thesaurus. Code C77818.

A person having origins in the any of the countries of South America or Central America who identifies as white. 